\title{
ON MODULI SPACES OF SYMPLECTIC FORMS
}

\author{
IVAN SMITH
}

\begin{abstract}
We prove that there are simply-connected four-manifolds which admit $n$-tuples of symplectic forms whose first Chern classes have pairwise different divisibilities in integral cohomology. It follows that the moduli spaces of symplectic forms modulo diffeomorphisms on the manifolds are disconnected.
\end{abstract}

\section{Introduction}

This paper provides examples of symplectic manifolds with disconnected moduli spaces of symplectic forms. Recall that two symplectic forms $\omega$ and $\omega^{\prime}$ on a symplectic manifold $X$ are deformation equivalent if there is some path of symplectic forms interpolating between them. We allow the cohomology class to vary both along the path and at the endpoints. The forms $\omega$ and $\omega^{\prime}$ are diffeomorphism related if there is some diffeomorphism $\phi: X \rightarrow X$ for which $\phi^{*} \omega=\omega^{\prime}$; we do not insist that $\phi$ is isotopic to the identity. We write $\mathcal{M}(X)$ to denote the moduli space of symplectic forms on $X$ up to diffeomorphism.

Definition 1.1. The forms $\omega$ and $\omega^{\prime}$ are equivalent symplectic forms on $X$ if they lie in the same equivalence class under the equivalence relation generated by deformations and diffeomorphisms.

Thus equivalence classes of symplectic form are indexed by $\pi_{0}(\mathcal{M}(X))$. This is a suitable notion of equivalence for forms when we are interested in essentially topological properties of the underlying symplectic manifolds. Note that even coarse symplectic invariants of $X$, such as total volume, are not preserved by an equivalence of forms.

In dimensions $4 k$ with $k>1$ there are many examples of manifolds with inequivalent symplectic forms, which can be distinguished by the divisibility of the first Chern class in integral cohomology. (In [8], using Gromov-Witten invariants, Yong-Bin Ruan gave sophisticated examples of inequivalent forms with the same Chern classes - on algebraic 3-folds; notice that he uses the term "deformation equivalent" for our "equivalent". Ruan also gave examples [7] of diffeomorphisms of 3-folds which do intertwine Chern classes but which do not induce an equivalence of forms.) To see this, note that given homeomorphic fourmanifolds $X$ and $Y$, the products $X \times X=Y \times Y=Z$ are diffeomorphic. Suppose $X$ is a minimal complex surface of general type with first Chern class divisible by any odd $n>1$, and $Y$ is a non-minimal symplectic four-manifold

Received September 13, 2000. 
homeomorphic to $X$. (It is straightforward to construct such pairs using Freedman's theorems.) The product symplectic form $\omega_{X} \oplus \omega_{X}$ on $Z$ has odd $c_{1}$ whereas the form $\omega_{Y} \oplus \omega_{Y}$ has $c_{1}$ prime. Taking additional products with further copies of the manifolds extends this to all higher dimensions $4 k$. However, in dimension four until recently no manifolds with inequivalent symplectic forms were known. This question was settled by McMullen and Taubes in [6], but the forms were distinguished by gauge theory techniques specific to four dimensions. In this paper we provide four-dimensional examples on a similar footing to the higher dimensional examples sketched above. Our main result is the following

Theorem 1.2. For each $n \geq 2$ there is a simply-connected manifold $X^{4}$ which admits $n$ distinct symplectic forms whose first Chern classes are of pairwise different divisibilities in $H^{2}(X ; \mathbb{Z})$. In particular for such an $X$ we have $\left|\pi_{0}(\mathcal{M}(X))\right| \geq n$.

We note that a recent result of $\mathrm{Li}$ and $\mathrm{Liu}$ [4] shows that on any symplectic fourmanifold, there are at most finitely many orbits of the diffeomorphism group on the set of first Chern classes of symplectic structures. In some sense the above result is therefore optimal. (In contrast to the work of Ruan mentioned above, the existence of inequivalent symplectic forms not distinguished by the first Chern class remains open in four dimensions.) Theorem (1.2) perhaps casts a new light, via taking products, on a conjecture of Donaldson (1.3 and 1.4). The proof shall require only elementary arguments. Before giving more details, we set the result in context.

- Moduli spaces of symplectic forms: Following work of Taubes relating Seiberg-Witten invariants to pseudoholomorphic curves, there are a few symplectic four-manifolds for which the moduli space of symplectic forms is entirely understood. In all such cases, the understanding comes from a uniqueness theorem: for instance, every symplectic form on $\mathbb{C P}^{2}$ is standard up to scale and diffeomorphism [11]. Lalonde and McDuff have proved similar results for other rational and ruled manifolds [3]. Moreover in four dimensions many symplectic invariants are largely determined by the smooth structure of the underlying manifold, most famously for certain Gromov invariants counting pseudoholomorphic curves [11]. Recently McMullen and Taubes [6] gave the first examples of symplectic four-manifolds for which there are symplectic forms which are not equivalent to each other under any composition of deformations and pullbacks by diffeomorphisms. Their examples were distinguished by a classical invariant of a symplectic structure, the first Chern class, but via non-classical methods. First Chern classes of symplectic structures on manifolds with $b_{+}>1$ are always Seiberg-Witten basic classes. Diffeomorphisms of a manifold preserve the set of basic classes inside $\mathbb{R}^{b_{2}}=H^{2}(X ; \mathbb{R})$; since diffeomorphisms act on this space linearly, they preserve the polytope which is the convex hull of the basic classes. It follows that they must preserve the sets of vertices of this polytope of any fixed valence; McMullen and Taubes find two symplectic structures on a fixed manifold for which the first Chern classes represent vertices of distinct valence. 
Detecting the non-transitivity of diffeomorphisms on basic classes is of great interest in its own right, but it is natural to hope that one might distinguish symplectic structures on simply connected manifolds using purely classical methods. When a manifold contains a Lagrangian torus of square zero which is homologically essential, there are perturbations of the symplectic form making the torus a symplectic submanifold with either choice of orientation. This flexibility, which underlies the McMullen-Taubes construction in different language, will enable us to give new and easy examples of simply connected four-manifolds for which the moduli space of symplectic forms is disconnected. (Embedded surfaces of square zero were also used in [9] to give a topological construction of exotic symplectic submanifolds on complex surfaces.)

- Donaldson's Conjecture: According to ([5]; p.437) Simon Donaldson has formulated the following remarkable conjecture relating diffeomorphisms of symplectic four-manifolds to equivalences of symplectic six-manifolds. Write $\omega_{s t}$ for the standard Kähler form on projective space.

Conjecture 1.3. Let $\left(X, \omega_{X}\right)$ and $\left(Y, \omega_{Y}\right)$ be symplectic four-manifolds which are homeomorphic. Then they are diffeomorphic if and only if the product symplectic forms $\omega_{X} \oplus \omega_{\text {st }}$ and $\omega_{Y} \oplus \omega_{\text {st }}$ are equivalent on $X \times \mathbb{S}^{2} \cong_{\mathrm{diff}} Y \times \mathbb{S}^{2}$.

We stress that we have nothing to say about this directly; however, it follows from our examples that the sphere $\mathbb{S}^{2}$ is playing a special role here which is not immediately apparent from the formulation of (1.3). From (1.2) we see

Corollary 1.4. If in the statement of Donaldson's conjecture one replaces $\mathbb{S}^{2}$ by a symplectic manifold with $c_{1}$ divisible by $n>2$, then the analogous conjecture fails.

Hence we cannot replace $\mathbb{S}^{2}$ by the torus $\mathbb{T}^{2}$ or the $K 3$ surface. A more vivid statement is provided by the following contrast. Donaldson's conjecture would imply that the obvious symplectic structures on $X \times\left(\mathbb{P}^{1} \times \mathbb{P}^{1}\right)=Y \times\left(\mathbb{P}^{1} \times \mathbb{P}^{1}\right)$ are equivalent, whereas (1.4) shows that if we replace $\mathbb{P}^{1} \times \mathbb{P}^{1}$ with $\operatorname{Sym}^{2}\left(\mathbb{P}^{1}\right) \cong \mathbb{C P}^{2}$ then this need not be true.

The heart of (1.2) is the following sufficient condition for a four-manifold to admit inequivalent symplectic forms. Write $E(1)$ for the rational elliptic surface $[2]$.

Theorem 1.5. Let $X$ be a symplectic four-manifold such that

1. there are square-zero tori $T_{1}, \ldots, T_{r} \subset X$ which span an $r$-dimensional subspace in $H_{2}(X ; \mathbb{R})$, for some $r>1$;

2. $T_{1}$ is represented by a Lagrangian submanifold, $T_{i>1}$ and $\sum_{i} T_{i}$ are represented by symplectic submanifolds (tori), all disjointly embedded;

3. the first Chern class $c_{1}(X)$ is divisible by $n$ for some $n>2$, whilst ${ }^{1} n V 2 d$ where $d$ is the divisibility of $\left[T_{1}\right] \in H_{2}(X ; \mathbb{Z})$.

\footnotetext{
${ }^{1}$ We use the symbol $a \bigvee b$ to show that $a$ does not divide $b$.
} 
Then the manifold $Z$ given by fibre summing $X$ with a copy of $E(1)$ along each of $T_{1}, \ldots, T_{r}$ and along $(n-1)$ parallel copies of $T_{1}+\cdots+T_{r}$ admits inequivalent symplectic structures.

The smooth structure on $Z$ depends on a choice, once and for all, of trivialisations of the normal bundles of the tori. If $\pi_{1}(X)$ is normally generated by the images of the $\pi_{1}\left(T_{i}\right)$ then $Z$ is simply connected. Here is an example. Let $\mathbb{T}^{4}$ describe the usual Euclidean four-torus $\mathbb{R}^{4} / \mathbb{Z}^{4}$. With co-ordinates $x, y, z, t$ on $\mathbb{R}^{4}$ there are two-tori in $\mathbb{T}^{4}$ which come from projecting the planes described by the pairs of lines

$$
\langle x, t\rangle ;\langle y, t\rangle ;\langle z, t\rangle ;\langle x=y=z, t\rangle .
$$

Call these respectively $T_{x}, T_{y}, T_{z}, T_{w}$. All the tori are oriented by taking the usual orientations on the defining lines in $\mathbb{R}^{4}$ (pointing out towards $+\infty$ ). We can perturb the tori to obtain five disjoint oriented tori inside $\mathbb{T}^{4}$; two parallel copies of $T_{w}$ and one of each of $T_{x}, T_{y}, T_{z}$. Let $Z$ denote the simply connected smooth four-manifold obtained by forming the normal fibre sum of $\mathbb{T}^{4}$ with five copies of $E(1)$, gluing a standard complex fibre $F \subset E(1)$ to each of the five tori above.

Corollary 1.6. The simply-connected manifold $Z$ admits two symplectic structures which are not equivalent under any sequence of deformations or diffeomorphisms. Indeed there are symplectic structures $\omega_{1}$ and $\omega_{2}$ on $Z$ for which $c_{1}\left(T Z ; \omega_{1}\right)$ is divisible by 3 in integral cohomology but $c_{1}\left(T Z ; \omega_{2}\right)$ is prime.

Since the construction allows for some variation, infinitely many homeomorphism types of simply connected four-manifold displaying a similar phenomenon can be obtained. To obtain a manifold with $N$ inequivalent symplectic forms, take $n=p_{1} \cdots p_{N}$ to be a product of $N$ distinct odd primes in (1.5). Then by varying the number of fibre sums performed with the "wrong" orientation along the Lagrangian torus, one can obtain symplectic forms on the final manifold $Z$ for which the first Chern class is divisible by precisely one of the primes $p_{i}$. Thus (1.2) will be a direct consequence of the above results. We will address the proofs of (1.5 and 1.6) in the subsequent sections. Using these, and the remarks at the end of the paper, it is straightforward to deduce (1.4); we leave the details to the reader.

\section{Lagrangian fibre sums}

The fibre-sum construction for symplectic manifolds was pioneered by Gompf in [1] who used it to construct symplectic manifolds displaying many new phenomena. Gompf also noticed that given a homologically essential Lagrangian submanifold $L$ of a symplectic four-manifold $X$, one could perturb the ambient symplectic form $\omega$ by an arbitrarily small amount so as to make the form non-vanishing on $L$. In the presence of a fixed orientation on $L$, there is some choice here; one can perturb $\omega$ to be either positive or negative on $L$. Recently this construction, viewed from a somewhat different perspective, has been used 
by McMullen and Taubes to produce symplectic manifolds with inequivalent symplectic forms. Their examples were distinguished using Seiberg-Witten theory, and the action of a diffeomorphism of a four-manifold on the convex hull of the Seiberg-Witten basic classes. Via the description in terms of fibre sums along Lagrangians, one can obtain simpler examples of the same phenomena; we present one class here. The author is very grateful to Ron Fintushel for a lucid explanation of the original work of McMullen and Taubes.

We begin with some more general remarks on the fibre sum operation in our context. Let $(X, T)$ and $\left(Y, T^{\prime}\right)$ be symplectic pairs, where $X, Y$ are symplectic four-manifolds and $T, T^{\prime}$ are embedded tori of square zero on which the symplectic forms are non-degenerate. The symplectic structures define orientations on all four manifolds, and hence orientations on the normal bundles of $T$ in $X$ and $T^{\prime}$ in $Y$. Then given any orientation-preserving diffeomorphism $f$ of $T$ and $T^{\prime}$, we may lift to an orientation-reversing diffeomorphism of the normal bundles and use this to glue the manifolds $X$ and $Y$ along a neighbourhood of the embedded surfaces. Explicitly, choose a marking of $T$ and $T^{\prime}$ with a standard torus so the diffeomorphism $f$ becomes the identity. Then we form

$$
X \sharp_{\left(T: T^{\prime}\right)} Y=X \backslash \nu_{X} T \cup_{\tau} Y \backslash \nu_{Y} T^{\prime}
$$

where $\tau: T \times D^{2} \rightarrow T^{\prime} \times D^{2}$ is the map taking $(t, z) \mapsto(t, \bar{z})$. Since complex conjugation reverses orientation on the disc and on its boundary circle, there is a natural orientation induced on the final manifold. The diffeomorphism type of the final manifold depends a priori on the diffeomorphism $f$ and the choice of lift to an identification of the normal bundles. In our applications, the normal bundles of the tori will be canonically trivial. If in addition we suppose that $\left(Y, T^{\prime}\right)$ is an elliptic surface $(E(1), F)$ with an embedded complex fibre, then in fact all choices of $f$ give orientation-preserving diffeomorphic manifolds. This is because of the standard

Lemma 2.1. Let $E(1)$ denote the complex elliptic surface given by blowing up the nine base-points of a generic pencil of cubics in $\mathbb{C P}^{2}$. Let $F$ denote a generic smooth complex fibre of the resulting elliptic fibration. Then every orientation preserving diffeomorphism of $\nu_{E(1)} F$ may be extended to an orientation preserving diffeomorphism of $E(1)$.

Note importantly that in this case, the smooth structure on the fibre sum is determined completely by the orientation on $T$ and by the trivialisations of the normal bundles. The orientation of the torus in $E(1)$ is not important since there are diffeomorphisms of the boundary of a neighbourhood of the fibre which are orientation preserving but reverse the orientation on the fibre. Once all the choices for the smooth structure on the sum are fixed, we can consider how to patch forms. That is, the symplectic structures themselves enter only in putting a symplectic form on the sum.

We will make regular use of the following: 
Proposition 2.2. Let $W=X_{\left(_{\left(T: T^{\prime}\right)}\right.} Y$ denote the symplectic sum of manifolds $X, Y$ along embedded symplectic tori of square zero. Then

$$
c_{1}(W)=c_{1}(X)+c_{1}(Y)-2 \mathcal{P}\left(\left[T=T^{\prime}\right]\right) .
$$

Here $\mathcal{P}(\cdot)$ denotes the Poincaré dual of a homology class (we will suppress this from the notation henceforth).

Proof. The notation needs explanation, since $c_{1}(X)$ and $c_{1}(Y)$ are not naturally elements of $H^{2}(W)$. One can make sense of them in various ways. Suppose inside $X$ and $Y$ we have embedded surfaces which are representatives for $c_{1}$ disjoint from the tori $T$ and $T^{\prime}$. In this case these surfaces define homology classes in the manifold $W$ and we take the Poincaré duals of these. The tangent bundle to an oriented torus is (canonically) trivial, so the canonical bundles of $X$ and $Y$ are trivial over neighbourhoods of $T$ and $T^{\prime}$. Hence we can obtain the surfaces we require by choosing smooth sections of $K_{X}^{-1}$ and $K_{Y}^{-1}$ which are constant and non-vanishing near $T$ and $T^{\prime}$.

There is apparently some choice. The trivialisations of the canonical bundles near the tori depend on a choice of trivialisation of the normal bundles of the tori. For our later examples there are natural choices: the fibre in an elliptic fibration has a canonically trivial normal bundle, whilst any Lagrangian torus has a canonically trivial normal bundle since a (connected) choice of almost complex structure defines an isomorphism from the normal bundle to the tangent bundle. Being more careful, recall that

$$
H_{2}\left(X \backslash \nu_{X}(T)\right)=H^{2}\left(X \backslash \nu_{X}(T) ; \partial\right) ;
$$

one can see that a choice of trivialisation of the normal bundle to $T$ defines a relative Chern class in the group on the right. Moreover, we can use the given trivialisation of $\nu_{X}(T)$ and diffeomorphism $T \rightarrow T^{\prime}$ to define one for $\nu_{Y}\left(T^{\prime}\right)$, hence a second relative Chern class in $H^{2}\left(Y \backslash \nu_{Y}\left(T^{\prime}\right) ; \partial\right)$. Perform the gluing relative to these normal trivialisations; changing the trivialisation affects the two relative Chern classes in cancelling fashion. Thus the final result is independent of choices.

Interpreting the statement of the Proposition appropriately, a proof runs as follows. We can choose smooth sections of the anticanonical bundles of each of $X, Y$ which are non-vanishing over a neighbourhood of the gluing tori. Then the final Chern class differs from the sum by a contribution from the normal directions to the tori. The tangent bundles to $X$ and $Y$ split as a product here, and the result follows from the usual formula for connect summing twodimensional surfaces:

$$
e\left(\Sigma_{1} \sharp \Sigma_{2}\right)=e\left(\Sigma_{1}\right)+e\left(\Sigma_{2}\right)-2 .
$$

Equivalently, the result follows since complex conjugation on the annulus has algebraically two negatively oriented fixed points; compare to a favourite Kähler example. 
Now suppose $(X, T)$ is a pair comprising a symplectic four-manifold and an oriented Lagrangian torus $T$, which we suppose is non-trivial in homology. Gompf [1] notes that we can perturb the symplectic form $\omega_{X}$ (by an arbitrarily small amount, and in particular preserving the first Chern class of the symplectic structure) to be non-degenerate on $T$. Fixing a pair of oriented tangent vectors $v_{1}, v_{2}$ to $T$ at a point $p \in T$, we then have two possibilities: $\omega_{X}\left(v_{1}, v_{2}\right)_{p}>0$ or $\omega_{X}\left(v_{1}, v_{2}\right)_{p}<0$. That is, the symplectic orientation induced on $T$ can agree or disagree with the given fixed orientation. In the first case, we can form the symplectic sum of $(X, T)$ with $(E(1), F)$ in the usual way, and we will find that (writing $T_{+}$to note the positivity)

$$
c_{1}\left(X \sharp_{\left(T_{+}: F\right)} E(1)\right)=c_{1}(X)+c_{1}\left(E(1) ; \omega_{0}\right)-2\left[T_{+}=F\right]
$$

comparing with (2.2). Here $\omega_{0}$ denotes the usual Kähler form on the rational elliptic surface. However, for a perturbation of $\omega_{X}$ for which $T$ is negatively oriented, if we form the fibre sum of $X$ along $T$ with the given and not symplectic orientation for the normal bundle $\nu_{T / X}$, then we can only sum with a pair $\left(Y, T^{\prime}\right)$ for which

$$
\int_{T^{\prime}} \omega_{Y}=\int_{T} \tilde{\omega}_{X}<0
$$

where the $\tilde{r}$ denotes the perturbed form. Taking $Y=E(1)$ and $T^{\prime}=F$ we find that we can now form the symplectic sum if we take $-\omega_{0}$, the negative of the usual Kähler form, as the symplectic form on $E(1)$. Then looking at Chern classes we find

$$
c_{1}\left(X \sharp_{\left(T_{-}: F\right)} E(1)\right)=c_{1}(X)+c_{1}\left(E(1) ;-\omega_{0}\right)-2\left[-T_{-}=-F\right] .
$$

Since we are fibre summing along surfaces which have the reversed orientation to the usual symplectic setting (2.2), in the last term we reverse sign. The following is standard:

Lemma 2.5. For the rational elliptic surface we have

$$
c_{1}\left(E(1) ; \omega_{0}\right)=[F], \quad c_{1}\left(E(1) ;-\omega_{0}\right)=-[F]
$$

identifying homology and cohomology via Poincaré duality as usual.

With these various comments in place, the theorem (1.5) is straightforward.

Theorem 2.6. Suppose $X$ is a symplectic manifold as in the statement of (1.5). Pick an orientation on $T_{1}$ and form the oriented fibre sum of $X$ with $r+n-1$ copies of $E(1)$ as described. Then the resulting manifold $Z$ admits inequivalent symplectic structures. Moreover it is simply connected if $\pi_{1}(X)$ is normally generated by the images of the $\pi_{1}\left(T_{i}\right)$.

Proof. We put two symplectic structures on $Z$, the first by perturbing $T_{1}$ to have the positive symplectic orientation and summing with $\left(E(1), \omega_{0}\right)$ and the second by perturbing in the other direction and summing with $\left(E(1),-\omega_{0}\right)$. From the 
above lemmata we find that the two symplectic structures on $Z$ have Chern classes

$$
c_{1}(Z)_{+}=c_{1}(X)-\left[T_{1}\right]-\left[T_{2}\right]-\cdots-\left[T_{r}\right]-(n-1)\left[T_{1}+\cdots+T_{r}\right]
$$

in the positive case, and

$$
c_{1}(Z)_{-}=c_{1}(X)+\left[T_{1}\right]-\left[T_{2}\right]-\cdots-\left[T_{r}\right]-(n-1)\left[T_{1}+\cdots+T_{r}\right]
$$

in the negative case. Since $n \mid c_{1}(X)$ clearly $n \mid c_{1}\left(Z ; \omega_{+}\right)$; however, $c_{1}\left(Z ; \omega_{-}\right)=$ $n A+2\left[T_{1}\right]$ for $A=\left(c_{1}(X) / n\right)-\sum\left[T_{i}\right]$. Recalling that $n \vee 2 d$ for $d$ the divisibility of $\left[T_{1}\right]$, the Chern class $c_{1}(Z)_{-}$cannot be divisible by $n$, and the result follows. The statement on fundamental groups is straightforward since $\pi_{1}(E(1) \backslash F)=0$.

In the last section we provide some explicit examples. For simplicity, note that the divisibility condition $n V 2 d$ will always hold if $\left[T_{1}\right]$ is prime in homology, whilst $n \mid c_{1}(X)$ is trivial for any $n$ if $c_{1}(X)=0$.

\section{Inequivalent symplectic forms}

We develop the examples described in (1.6). The reader may wish to compare to the original construction of McMullen and Taubes from [6]. Take co-ordinates $x, y, z, t$ on $\mathbb{R}^{4}$ and write $\mathbb{T}^{4}=\mathbb{R}^{4} / \mathbb{Z}^{4}$. Define the oriented tori $T_{x}, T_{y}, T_{z}$ and $T_{w}$ as follows:

$$
T_{x}=\langle x, t\rangle ; T_{y}=\langle y, t\rangle ; T_{z}=\langle z, t\rangle ; T_{w}=\langle x=y=z, t\rangle
$$

These tori are oriented as follows. View $\mathbb{T}^{4}$ as $\mathbb{T}^{3} \times \mathbb{S}^{1}$ and view the tori as given by taking products of circles in $\mathbb{T}^{3}$ with the last factor. The circles are projections of lines in $\mathbb{R}^{3}$ which are all disjoint and are parallel to the $x, y, z$ axes and to the line $x=y=z$ respectively. Orient these lines by arrows pointing to the positive infinity, and orient the circle $\mathbb{S}_{(t)}^{1}$; this orients the tori. We choose the lines in $\mathbb{R}^{3}$ parallel to the obvious axes so that all the tori are disjoint.

Lemma 3.2. There is a relation $\left[T_{w}\right]=\left[T_{x}+T_{y}+T_{z}\right]$ in $H_{2}\left(\mathbb{T}^{4} ; \mathbb{Z}\right)$.

Proof. This is straightforward from the given presentation. We recall for the reader's convenience, however, the way this comes about via the original construction. From [6]: View $\mathbb{T}^{3}$ as surgery on the Borromean rings $\mathcal{B}$ in $\mathbb{R}^{3}$. The circles $C_{x}, C_{y}, C_{z}$ in $\mathbb{T}^{3}$ for which $C_{\bullet} \times \mathbb{S}^{1}=T_{\bullet}$ are the images of meridians to the three zero-framed components of the link $\mathcal{B}$. On the other hand, the circle $C_{w}$ is the image of an axis to the link in $\mathbb{R}^{3}$, that is a line linking each component of the rings precisely once. The meridians $C_{x}, C_{y}, C_{z}$ generate $H_{1}\left(\mathbb{T}^{3} ; \mathbb{Z}\right)$ and the co-ordinates of any loop $\gamma$ in $H_{1}$ with respect to this basis are given by the linking numbers, which yields the formula claimed. 
From this construction, or directly, we can take two parallel and unlinked copies of the axis in $\mathbb{R}^{3}$ and obtain two parallel copies of the torus $T_{w} \subset \mathbb{T}^{4}$ each of which satisfies this homology relation. For the standard symplectic structure on $\mathbb{T}^{4}$ induced from $d x \wedge d t+d y \wedge d z$ on $\mathbb{R}^{4}$, the tori $T_{x}$ and $T_{w}$ are symplectic whilst the tori $T_{y}, T_{z}$ are Lagrangian (but canonically oriented by the orientations on the axes in $\mathbb{R}^{3}$ ). By the remark after (2.1), there is a uniquely defined oriented smooth four-manifold $Z$ given by forming the fibre sum of $\mathbb{T}^{4}$ with five copies of $E(1)$. Here we glue a neighbourhood of a complex oriented fibre $F \subset E(1)$ to the oriented tori $T_{x}, T_{y}, T_{z}$ and to two parallel copies of $T_{w}$. Note that all the tori have canonically trivial normal bundles, and this fixes the indeterminacy in the fibre sum. Because $\pi_{1}\left(\mathbb{T}^{4}\right)$ is generated by the images of the fundamental groups $\pi_{1}\left(T_{\bullet}\right) \rightarrow \pi_{1}\left(\mathbb{T}^{4}\right)$, and because $\pi_{1}(E(1) \backslash F)$ is trivial (contract any loop along a cusp fibre, which is a rational curve), it follows easily that $Z$ is simply connected. Note that the McMullen-Taubes manifold of [6] is exactly the smooth fibre sum of $\mathbb{T}^{4}$ along the four oriented tori $T_{x}, T_{y}, T_{z}$ and one copy of $T_{w}$. Using our remarks on Chern classes, we have the following proposition, from which the theorem (1.6) clearly follows.

Proposition 3.3. The homology classes $-3\left[T_{x}+T_{y}+T_{z}\right]$ and $-3\left[T_{x}+T_{y}\right]-T_{z}$ are each the first Chern class of a symplectic structure on $Z$.

Proof. There is a symplectic structure $\omega_{+}$on $\mathbb{T}^{4}$ for which all four tori are symplectic submanifolds with the symplectic orientation and given orientations agreeing. Form the symplectic fibre sums with respect to such a form, inducing a form $\Omega_{+}$on $Z$. Then we find by $(2.3)$ that $c_{1}\left(Z ; \Omega_{+}\right)$is given by

$$
\begin{gathered}
\sum_{i=1}^{5} c_{1}\left(\mathbb{T}^{4} ; \omega_{+}\right)+\left[F_{x}+F_{y}+F_{z}+F_{w}+F_{w}\right] \\
-2\left[F_{x}=T_{x}\right]-2\left[F_{y}=T_{y}\right]-2\left[F_{z}=T_{z}\right]-2\left[F_{w}=T_{w}\right]-2\left[F_{w}=T_{w}\right],
\end{gathered}
$$

where we write $F_{\bullet}$ for the homology class of the fibre in the - th copy of $E(1)$ for clarity. However, we can also choose a form $\omega_{-}$on $\mathbb{T}^{4}$ for which $T_{x}, T_{y}$ and $T_{w}$ are symplectic with the given orientation but for which $\omega_{-}$restricts on $T_{z}$ to a form which is symplectic with the negative orientation. Now use (2.4) to compute the Chern class of the symplectic form $\Omega_{-}$induced on $Z$ after performing the new symplectic sum (which is smoothly identical to the old). Recall from (2.5) that the sign of the first Chern class of $E(1)$ changes if we change the sign of the symplectic form: then $c_{1}\left(Z ; \Omega_{-}\right)$is now given by

$$
\begin{gathered}
\sum_{i=1}^{5} c_{1}\left(\mathbb{T}^{4} ; \omega_{-}\right)+\left[F_{x}+F_{y}-F_{z}+F_{w}+F_{w}\right] \\
-2\left[F_{x}=T_{x}\right]-2\left[F_{y}=T_{y}\right]+2\left[F_{z}=T_{z}\right]-2\left[F_{w}=T_{w}\right]-2\left[F_{w}=T_{w}\right] .
\end{gathered}
$$

Now recall that for both $\omega_{ \pm}$the first Chern class of $\mathbb{T}^{4}$ vanishes, since these are perturbations of the standard form; then expanding out and using (3.2) the proposition follows. 
One can fibre sum with further parallel copies of $T_{w}$ to obtain a sequence of (pairwise non-homeomorphic) simply connected manifolds with pairs of symplectic structures, precisely one of which has $c_{1}$ divisible by $n$, for any $n>2$. If $n=p_{1} \cdots p_{N}$ is a product of primes and we perform $p_{i}$ of the fibre sums with the negatively oriented Lagrangian torus, we obtain a symplectic structure with first Chern class divisible by $p_{i}$ but not any other $p_{j \neq i}$. This gives the main theorem (1.2). For more variation, replace the four-torus with some twisted torus bundle over the torus which has a symplectic structure with vanishing first Chern class. Infinite families of such examples are described in [10].

\section{Acknowledgements}

The McMullen-Taubes construction was cast in the light presented here during a lecture by Ron Fintushel, and I am grateful to him for helpful correspondence. I am also indebted to Bob Gompf, who offered valuable comments on an earlier draft of the paper.

\section{References}

[1] R. Gompf, A new construction of symplectic manifolds, Ann. of Math. 142 (1995), 527595.

[2] R. Gompf and A. Stipsicz, 4-manifolds and Kirby calculus, Graduate Studies in Mathematics 20, American Mathematical Society, 1999.

[3] F. Lalonde and D. McDuff, J-curves and the classification of rational and ruled symplectic 4-manifolds, Contact and symplectic geometry (C.B. Thomas, ed.), Cambridge Univ. Press, 1996.

[4] T.-J. Li and A.-K. Liu, Uniqueness of symplectic canonical class, surface cone and symplectic cone of four-manifolds with $b_{+}=1$, preprint (2000).

[5] D. McDuff and D. Salamon, Introduction to symplectic topology (2nd edition) the Clarendon Press, Oxford University Press, 1998.

[6] C. T. McMullen and C. H. Taubes, 4-manifolds with inequivalent symplectic forms and 3-manifolds with inequivalent fibrations, Math. Res. Lett. 6 (1999), 681-696.

[7] Y. Ruan, Symplectic topology and extremal rays, Geom. Funct. Anal. 3 (1993), 395-430.

[8] _ Symplectic topology on algebraic 3-folds, J. Diff. Geom. 39 (1994), 215-227.

[9] I. Smith, Symplectic submanifolds from surface fibrations, Pacific J. Math. (1999), to appear.

[10] _ Torus fibrations on symplectic four-manifolds, Proceedings of the 7th Gokova Geometry and Topology conference, to appear.

[11] C. Taubes, The Seiberg-Witten and the Gromov invariants, Math. Res. Lett. 2 (1995), $221-238$.

Ivan Smith, New College, Oxford, OX1 3BN, England.

E-mail address: smithi@maths.ox.ac.uk 